\title{
ARTICLE \\ Soft electrostatic trapping in nanofluidics
}

\author{
Michael A. Gerspach ${ }^{1,2,3}$, Nassir Mojarad ${ }^{4, *}$, Deepika Sharma ${ }^{1,2,5}$, Thomas Pfohl ${ }^{1,3,6, \S}$ and Yasin Ekinci ${ }^{1,2}$
}

Trapping and manipulation of nano-objects in solution are of great interest and have emerged in a plethora of fields spanning from soft condensed matter to biophysics and medical diagnostics. We report on establishing a nanofluidic system for reliable and contact-free trapping as well as manipulation of charged nano-objects using elastic polydimethylsiloxane (PDMS)-based materials. This trapping principle is based on electrostatic repulsion between charged nanofluidic walls and confined charged objects, called geometry-induced electrostatic (GIE) trapping. With gold nanoparticles as probes, we study the performance of the devices by measuring the stiffness and potential depths of the implemented traps, and compare the results with numerical simulations. When trapping $100 \mathrm{~nm}$ particles, we observe potential depths of up to $Q \cong 24 k_{\mathrm{B}} T$ that provide stable trapping for many days. Taking advantage of the soft material properties of PDMS, we actively tune the trapping strength and potential depth by elastically reducing the device channel height, which boosts the potential depth up to $Q \sim 200 k_{\mathrm{B}} T$, providing practically permanent contactfree trapping. Due to a high-throughput and low-cost fabrication process, ease of use, and excellent trapping performance, our method provides a reliable platform for research and applications in study and manipulation of single nano-objects in fluids.

Keywords: contact-free electrostatic nanoparticle trapping; PDMS nanofluidic devices; high-throughput fabrication; single nano-object manipulation; soft-lithography

Microsystems \& Nanoengineering (2017) 3, 17051; doi:10.1038/micronano.2017.51; Published online: 4 December 2017

\section{INTRODUCTION}

The unique properties of the polydimethylsiloxane (PDMS) elastomer in making integrated microfluidic systems established its applications as a standard tool in a broad range of disciplines, such as disease diagnostics ${ }^{1}$, cell biology ${ }^{2}$, drug discovery ${ }^{3}$, and photonics ${ }^{4}$, to name a few. Biocompatibility, gas permeability, optically transparency, and having a low elastic modulus are its most important chemical and physical features that allow for its implementation in such diverse fields ${ }^{5}$. A reduction of channel dimensions and fabricating nanofluidic PDMS devices not only provides a higher efficiency and sensitivity to analytes, but could also allow for controlled processing of objects with nanometer dimensions in soft matter such as colloids, viruses and individual macromolecules. Recent efforts in developing tailored fabrication procedures led to the fabrication of nanofluidic PDMS channels that provide laminar flows without clogging or collapse ${ }^{6,7}$. More advanced integrated systems were also adapted to applications in, for example, protein preconcentration ${ }^{8}$, DNA stretching ${ }^{9}$, and Raman spectroscopy ${ }^{10}$.

However, stable trapping and manipulation of single nano-objects in PDMS devices are hindered by the lack of techniques that produce strong retraction forces acting against the driving force of randomization, the Brownian motion. On average, every single particle contains an energy of $1 / 2 k_{\mathrm{B}} T$ for each translational dimension. However, the instantaneous velocity of a particle follows a Maxwell-Boltzmann distribution ${ }^{11-13}$, in which the energy of the particle can temporarily considerably exceed the average value. To compensate for these high energy kicks, typically potential depths of around $10 k_{\mathrm{B}} T$ are required for stable trapping of an object in solution as established by Ashkin et al. ${ }^{14,15}$.

Geometry-induced electrostatic (GIE) trapping is an effective method for parallel trapping of charged nanoparticles in a nanofluidic system ${ }^{16}$. This method has shown to be efficient for levitating various types of objects including metal nanoparticles of various shapes ${ }^{17}, \operatorname{sizes}^{18,19}$ and charges ${ }^{20}$, as well as polystyrene beads $^{16}$ and lipid vesicles ${ }^{16,21}$. Moreover, it has been used in other applications such as single particle charge and size determination ${ }^{18}$, information storage ${ }^{22}$, and screening of electrostatic potentials ${ }^{23}$. The underlying physical principle of GIE-trapping is the creation of local electrostatic potential minima in a nanofluidic channel by introducing indentations in one surface of the channel. These indentations result in potential wells for stable trapping of nanoobjects carrying the same sign of the net charges as that of the walls (Figure 1a). For these devices, state-of-the-art fabrication procedures play a vital role, since smallest perturbations from the ideal device geometry largely distort the potential landscape. Hence, the fabrication of GIE-trapping devices has been limited to using $\mathrm{SiO}_{x}$ substrates processed by top-down nanofabrication procedures, making their production time consuming and resource demanding, practically limiting them to exploratory applications. As a result, PDMS-based GIE-trapping devices fabricated by soft lithography will substantially reduce production costs and time, making them easily to integrate into standard lab-on-a-chip systems, and making them available for research and commercial applications.

In this article, we report on the successful fabrication of PDMSbased nanofluidic systems that are used for GIE-trapping of

\footnotetext{
${ }^{1}$ Swiss Nanoscience Institute, Basel 4056, Switzerland; ${ }^{2}$ Laboratory for Micro and Nanotechnology, Paul Scherrer Institut, Villigen 5232 , Switzerland; ${ }^{3}$ Chemistry Department,

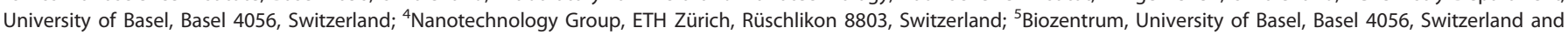
${ }^{6}$ Biomaterials Science Center, University of Basel, Allschwil 4123, Switzerland

Correspondence: Thomas Pfohl (thomas.pfohl69@gmail.com) and Yasin Ekinci (yasin.ekinci@psi.ch)

${ }^{*}$ Current address: ABB Switzerland Ltd., Semiconductors, Lenzburg 5600, Switzerland

${ }^{5}$ Current address: Institute of Physics, University of Freiburg, Freiburg 79104, Germany

Received: 22 April 2017; revised: 20 June 2017; accepted: 4 July 2017
} 


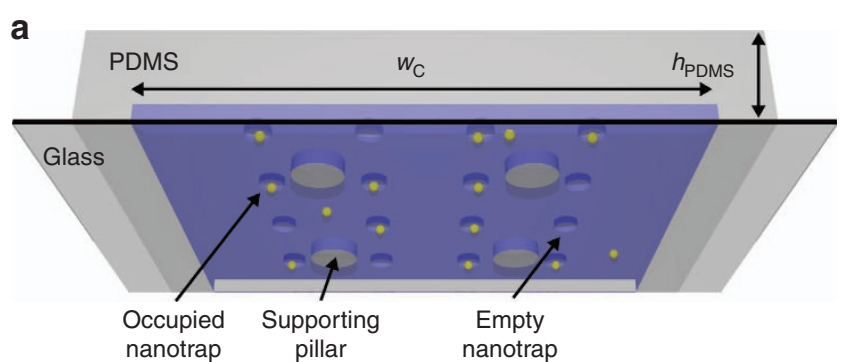

b

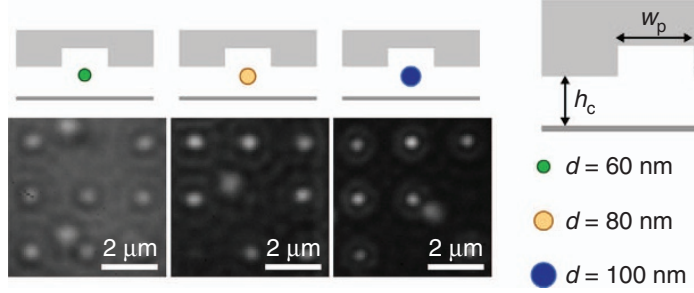

Figure 1 (a) Schematic of the polydimethylsiloxane (PDMS)-based nanofluidic trapping device with integrated pockets and supporting pillars. The nanofluidic channels had a width of $w_{c}=10 \mu \mathrm{m}$. Two device geometries $G_{1}\left(h_{\mathrm{c}}=210 \mathrm{~nm}, h_{\mathrm{p}}=70 \mathrm{~nm}\right)$ and $G_{2}\left(h_{\mathrm{c}}=160 \mathrm{~nm}\right.$, $h_{\mathrm{p}}=100 \mathrm{~nm}$ ) were used for trapping gold nanoparticles. The width $w_{p}$ of the pockets varied in both device geometries from 200 to $500 \mathrm{~nm}$. (b) Schematics and corresponding experimental optical iSCAT images of $d=60,80$ and $100 \mathrm{~nm}$ gold particles trapped in circular pockets with a diameter of $w_{p}=250 \mathrm{~nm}$ in $G_{2}$ devices. Scale bars: $2 \mu \mathrm{m}$.

charged nano-objects. Designing optimal device geometries, using precise fabrication techniques and an appropriate PDMS composition, preloading the device prior to bonding, and applying a tailored topographical design to avoid channel collapse play crucial roles in obtaining functional trapping devices. We use gold nanoparticles (Au NPs) down to $60 \mathrm{~nm}$ as probes to characterize the main trapping features such as the strength, lateral extension, and potential depth. For imaging, interferometric scattering detection (iSCAT) is used as a sensitive technique that allows for precise tracking of Au NPs as seen in Figure $1 b^{19,24}$. Beyond passive nanoparticle trapping, we also demonstrate active manipulation of the trap stiffness by locally applying a mechanical force to elastically deform the device, a feature that is not possible in $\mathrm{SiO}_{x}$-based devices. A successful implementation of PDMS nanofluidics would allow for facile production of functional nanofluidic systems and could provide a unique platform in fields such as single-molecule force spectroscopy, pharmaceutical drug discovery, and integrated labs-on-chips.

\section{MATERIALS AND METHODS \\ Device design}

The design of the multi-height GIE trapping silicon master is sketched in Figure 2a. It consists of two microfluidic reservoir channels and the nanofluidic GIE trapping region. The two microfluidic channels have a depth of $H=3 \mu \mathrm{m}$ and a width of $100 \mu \mathrm{m}$, each connected by an inlet and outlet. Several GIE trapping nanofluidic channels with a length of about $0.5 \mathrm{~mm}$ are connected with the microfluidic channels. Within these nanofluidic channels finer nanostructures, that is, the actual nanotraps are etched. The design schematics and fabrication steps of the nanofluidic trapping region are depicted in Figures 1,2 and 3. The nanofluidic channels have a width of $w_{c}=10 \mu \mathrm{m}$. Two device geometries were fabricated with a nanofluidic channel height of $h_{\mathrm{c}}=210 \mathrm{~nm}$ (device geometry $G_{1}$ ) or $h_{\mathrm{c}}=160 \mathrm{~nm}$ (device geometry $G_{2}$ ) as shown in Figure 2c. Along the width of each channel, circular indentations (pockets) with a depth of $h_{\mathrm{p}}=70 \mathrm{~nm}\left(G_{1}\right)$ or $h_{\mathrm{p}}=100 \mathrm{~nm} \quad\left(G_{2}\right)$ and diameters of $w_{\mathrm{p}}=200-500 \mathrm{~nm}$ were fabricated. Supporting pillars with a diameter of $1 \mu \mathrm{m}$ and a spacing of $4 \mu \mathrm{m}$ were implemented in the devices to prevent the channels from sagging and collapsing (Figures 1 and 2 and Supplementary Information S1). A final PDMS device filled with methylene blue for better visualization is shown in Figure $2 b$.

\section{Device fabrication}

The transition from micro- to nanofluidics requires advanced designs, material processing and handling techniques to obtain functional PDMS devices that are micrometer in width but only nanometers in height. The fabrication steps of the nanofluidic trapping region are shown in Figure 3. A silicon master was first made (Figure 3a) by top-down fabrication methods in a cleanroom facility, namely electron beam (e-beam) lithography, followed by reactive ion etching (RIE). Next, a replica molding was carried out using a UV curable hybrid polymer (OrmoStamp, micro resist technology $\mathrm{GmbH}, 12555$ Berlin, Germany) to obtain a negative copy of the original silicon master (Figure $3 b)^{25-27}$. This step brings along two major benefits: (i) the established fabrication steps available and optimized for silicon-based GIE trapping devices do not need a re-development to obtain negative masters for making PDMS devices, and (ii) replica molding into OrmoStamp results in high-throughput fabrication because several negative OrmoStamp masters can be produced from a single silicon master, which leads to benefits in rapid replication and possibilities for commercial applications (Figure 3d). The last step, transferring the OrmoStamp into PDMS (Sylgard 184 Silicone Elastomer, Dow Corning Corporation, Midland, 48686-0994 Michigan, USA) structures (Figure 3c) was then carried out under a laminar flowbox in a conventional chemistry lab. To increase the stiffness of PDMS and thereby reduce the risk of channel collapse $e^{28,29}$, PDMS was mixed at a crosslinking rate of 5:1 (prepolymer: crosslinker) resulting in an elastic modulus of about $E=3.6 \mathrm{MPa}^{30}$. The PDMS was cured on a hotplate at $150^{\circ} \mathrm{C}$, which is the optimized temperature for high patterning resolution ${ }^{31,32}$ and fast crosslinking. After curing, inlet and outlet reservoirs of $4 \mathrm{~mm}$ diameter were punched into the PDMS device as seen in Figure $2 \mathrm{~b}$. The detailed nanofabrication process is provided in the Supplementary Information S2.

\section{Sample solution preparation}

The Au NPs were purchased from BBI Solutions with a diameter $d$ of 60, 80, and $100 \mathrm{~nm}$ (EM.GC60/80/100, BBI Solutions, CF14 5DX Cardiff, UK). 60 and $80 \mathrm{~nm}$ Au NPs were centrifuged two times at $2000 \mathrm{~g}$. To exchange the buffer solution, the Au NP pellets were separated from the solution and re-suspended in fresh deionized (DI) water $(18 \mathrm{M} \Omega)$ each time. After a third centrifugation and extraction of the excess water, a dense solution of Au NPs of $\sim 10^{11}$ particles per $\mathrm{mL}$ was created. The extracted water was stored as a buffer solution to fill the microfluidic reservoirs and used to analyze the net charge of the particles and the ionic strength of the solution. To avoid clustering of the $100 \mathrm{~nm}$ Au NPs, centrifugation was done at $1500 \mathrm{~g}$ for $15 \mathrm{~min}$ two times and resuspended in DI water to exchange the buffer solution. A dense solution of about $10^{11}$ particles per $\mathrm{mL}$ of $100 \mathrm{~nm}$ gold particles was obtained after a third centrifugation step and decantation of the supernatant. The particle zeta potentials $\zeta_{\mathrm{p}}$, the solution conductivities $b$, and the diffusion coefficients $D$ were measured by phase analysis light scattering (Zetasizer Nano, Malvern Instruments, WR14 1XZ Malvern, UK). For 12 different measurements of each sample, 60, 80 and $100 \mathrm{~nm}$ Au NPs, the measured particle zeta potentials $\zeta_{\mathrm{p}}$ were $-36( \pm 2) \mathrm{mV},-34( \pm 2) \mathrm{mV}$ and -35 $( \pm 3) \mathrm{mV}$, the solution conductivities $b$ were $\sim 6.2 \mu \mathrm{sm}^{-1}$, $\sim 6.1 \mu \mathrm{S} \mathrm{cm}^{-1}$ and $\sim 5.5 \mu \mathrm{S} \mathrm{cm}^{-1}$, and the diffusion coefficient' $D$ were $\sim 6.4 \mu \mathrm{m}^{2} \mathrm{~s}^{-1}, \sim 5.2 \mu \mathrm{m}^{2} \mathrm{~s}^{-1}$ and $\sim 4.2 \mu \mathrm{m}^{2} \mathrm{~s}^{-1}$, 

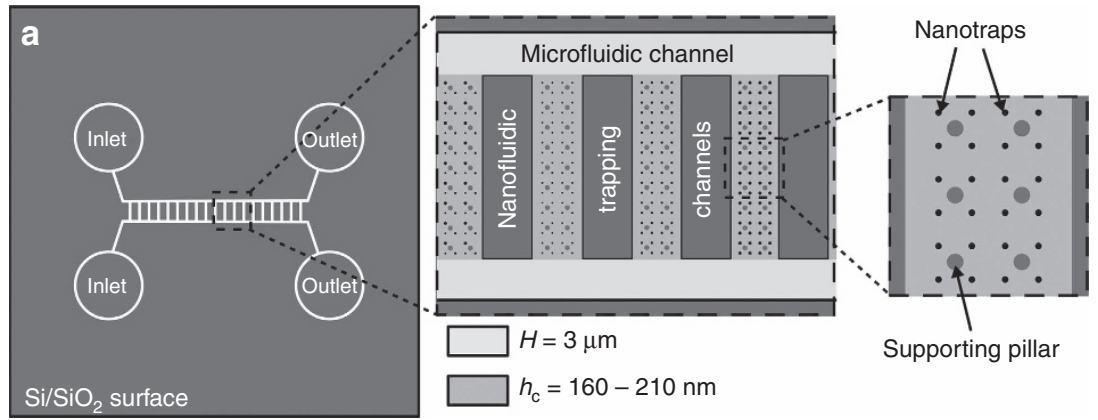

C

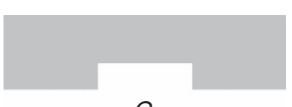

$G_{1}$

$$
\begin{aligned}
& h_{\mathrm{c}}=210 \mathrm{~nm} \\
& h_{\mathrm{p}}=70 \mathrm{~nm} \\
& w_{\mathrm{p}}=200-500 \mathrm{~nm}
\end{aligned}
$$
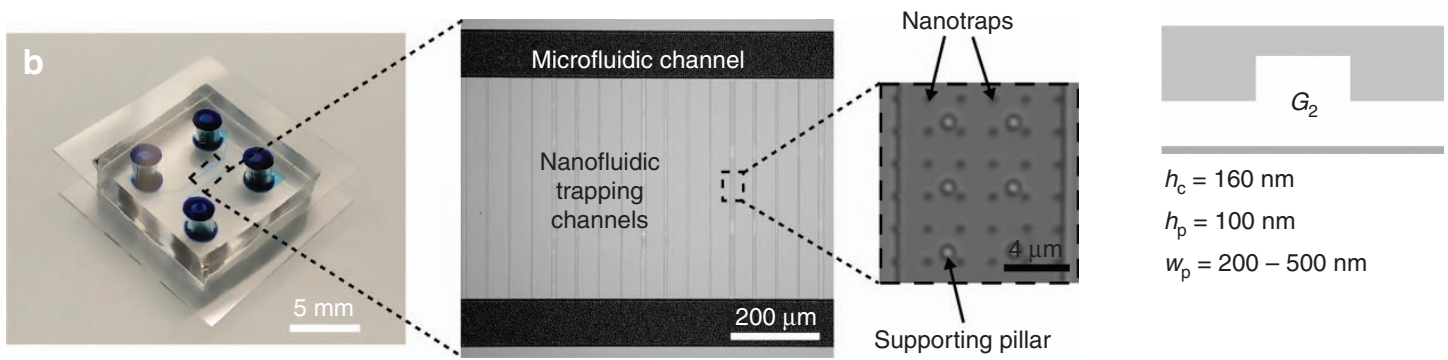

$h_{\mathrm{c}}=160 \mathrm{~nm}$

$h_{\mathrm{p}}=100 \mathrm{~nm}$

$w_{\mathrm{p}}=200-500 \mathrm{~nm}$

Figure 2 (a) Schematic of the silicon master design highlighting the two main features, the microfluidic reservoir channels and the nanofluidic geometry-induced electrostatic (GIE) trapping area. (b) Optical images of a finished polydimethylsiloxane (PDMS) device with the punched inlets and outlets and filled with a $0.2 \%$ methylene blue solution for better visualization of the microfluidic channels (left), a silicon master showing the magnified area of the microfluidic reservoir channels and the nanofluidic trapping channels (middle) and a silicon master showing one magnified nanofluidic trapping channel (right). Scale bars of images: left $5 \mathrm{~mm}$, middle $200 \mu \mathrm{m}$, right $4 \mu \mathrm{m}$. (c) Schematic of the two device geometries used for trapping gold nanoparticles.

respectively. From the measured zeta potentials and conductivities, the average surface charge density $\sigma_{\mathrm{p}}$ and particle net charges could be calculated using the semi-empirical equation ${ }^{18,33}$ $\sigma_{\mathrm{p}}=-\epsilon \epsilon_{0} K\left(k_{\mathrm{B}} T / j e\right)[2 \sinh (j y / 2)+(8 / \kappa d) \tanh (j y / 4)]$, with the permittivity of free space $\epsilon_{0}$, the dielectric constant of the medium $\epsilon$, the valence of the ions $j=1$, the Debye length ${ }^{34} K^{-1}=0.304 / \sqrt{c_{0}}$, and $y=\xi_{\mathrm{p}} e / k_{\mathrm{B}} T$ with the elementary charge $e$. The ionic strength of the solution of $0.09 \mathrm{mM}, 0.1 \mathrm{mM}$, and $0.1 \mathrm{mM}$ for each sample of 60, 80, and $100 \mathrm{~nm}$ Au NPs was estimated by the linear approximation $c_{0}=1.6 \times 10^{-2} b$, where $c_{0}$ is in units $\mathrm{mM}$ and $b$ in $\mu \mathrm{S} \mathrm{cm}^{-1}$ (Refs. 16,35). The particles net surface charge $q$ for 60 , 80 and $100 \mathrm{~nm}$ Au NPs was thus measured to be $\sim-92 e, \sim-168 e$ and $\sim-258 e$, corresponding to an average surface charge density of $\sigma_{\mathrm{p}} \sim 8 \times 10^{-3} \mathrm{e} \mathrm{nm}^{-2}$.

\section{Experimental procedure}

A 150- $\mu$ m-thick borofloat glass microscope coverslip (Borofloat 33, Plan Optik AG, 56479 Elsoff, Germany) was rinsed with acetone, IPA and DI water and dried by nitrogen blowing. The glass and a PDMS device were air-plasma activated for $35 \mathrm{~s}$ at a chamber pressure of $0.5 \mathrm{mbar}$ and $80 \%$ power (Femto, Diener electronic $\mathrm{GmbH}+\mathrm{Co}$. KG, 72224 Ebhausen, Germany). The PDMS parts were loaded prior bonding to the coverslip glass with a solution containing the nanoparticles to prevent collapsing during bonding ${ }^{29}$. Therefore, $0.25 \mu \mathrm{L}$ of the particle solution was placed directly on the nanofluidic channel region of the PDMS. Within $1 \mathrm{~min}$ after the activation, the PDMS device was gently pressed to the coverslip glass. After waiting another $1 \mathrm{~min}$ the PDMS was covalently bound to the coverslip and could not be separated anymore. Then about $60 \mu \mathrm{L}$ of the buffer solution was placed into each inlet, which filled the microfluidic reservoir channels by capillary forces. To stop the flow, $60 \mu \mathrm{L}$ of the buffer solution was filled into each outlet. Finally, the device was sealed by a second cover glass to avoid evaporation of the solutions as seen in Figure $2 \mathrm{~b}$. The finished device was placed on the microscope holder and the particles were recorded using the iSCAT setup.
For tuning the trap stiffness, the PDMS was compressed by an applied mechanical force. In particular, a precision screw (150$801 \mathrm{ME}$, Thorlabs Inc., Newton, NJ, USA) and a silicon plate of $4 \times 4 \mathrm{~mm}^{2} \times 0.5 \mathrm{~mm}$ was used. The pressure was applied to the PDMS surface by turning the screw clockwise to attain a stepwise deformation of the PDMS of about $\Delta L=15 \mu \mathrm{m}$ each, corresponding to a pressure increase of about $\triangle P=10 \mathrm{kPa}$. The deformation pressure was calculated by taking the Young's modulus $E=3.6 \mathrm{MPa}$ and the initial thickness of the PDMS of $h_{\mathrm{PDMS}}=5 \mathrm{~mm}^{30}$.

\section{Electron microscopy imaging and sample preparation}

To inspect if the nanostructures' morphology is preserved during the two-step replica molding transfer into OrmoStamp and PDMS, scanning electron microscopy (SEM) images were taken. To reduce charging effects during SEM imaging, a $15 \mathrm{~nm}$ chromium metal layer was sputtered on the OrmoStamp and the PDMS replica mold (Leica EM SCD 500, Leica Microsystems, 35578 Wetzlar, Germany, sputtering rate $0.1 \mathrm{~nm} \mathrm{~s}^{-1}$ ). The conductive silicon master was not specially pretreated. The wafers were imaged by a Zeiss Supra 55 VP SEM (Carl Zeiss AG, Jena, Germany) using the following imaging parameters: silicon master, EHT $10 \mathrm{kV}$, InLens, WD 6 mm; OrmoStamp master, EHT 1 kV, InLens, WD 5 mm; PDMS, EHT 3 kV, SE2, WD $16 \mathrm{~mm}$.

\section{Optical microscopy}

Interferometric scattering detection (iSCAT) was used as the imaging method for particle tracking ${ }^{16,24,36-39}$. The iSCAT signal is generated by the interference of a reference beam, which is reflected by a strongly reflecting interface in the device, and the beam scattered from the particle ${ }^{38,39}$. Similar to glass-based devices ${ }^{19}$, PDMS systems have a weak reference beam caused by having a refractive index close to that of water, which increases the detected signal-to-noise ratio (SNR) of the trapped objects (Supplementary Information S3). The setup was built using a $300 \mathrm{~mW}$ diode-pumped solid-state laser 

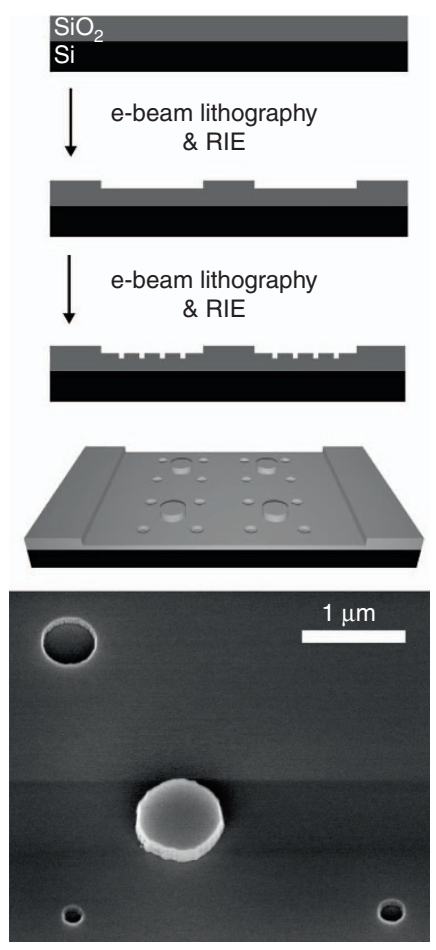

d

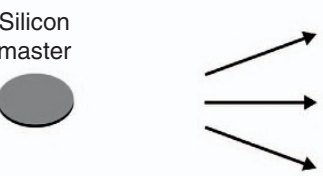

b
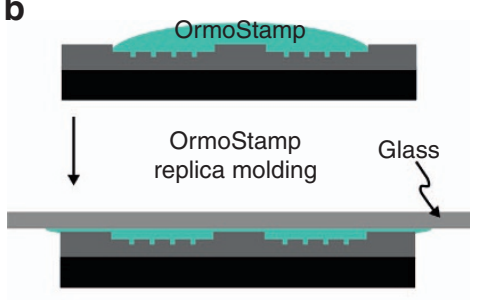

UV-cure

\& detachment
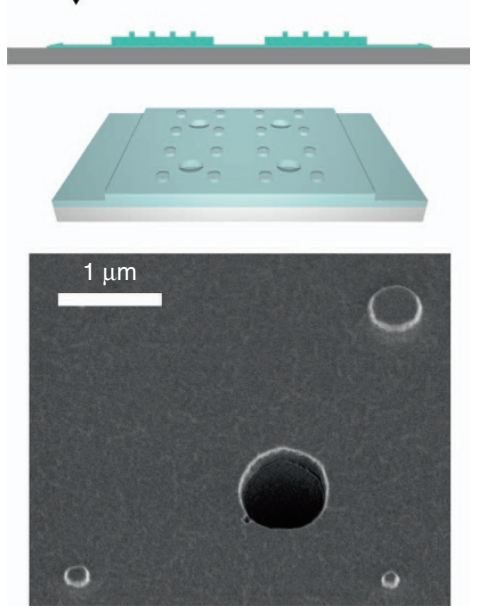

OrmoStamp masters
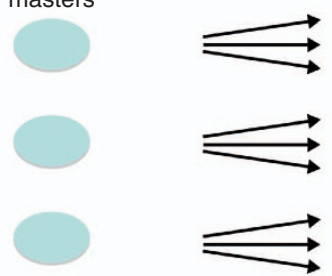

C
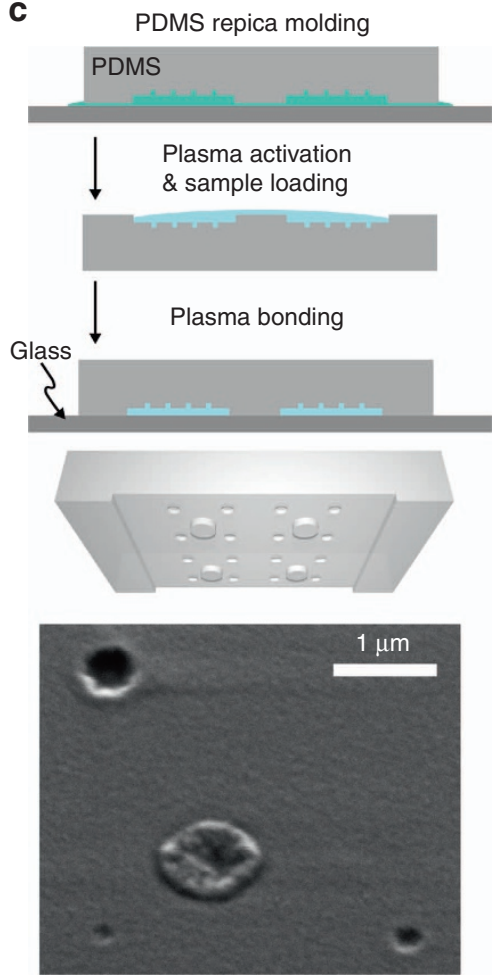

PDMS devices

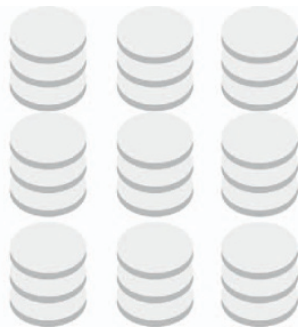

Figure 3 Side view sketch, three-dimensional (3D)-model and scaning electron microscope (SEM) images of the fabrication steps of the GIE trapping devices. Scale bars: $1 \mu \mathrm{m}$. (a) Fabrication of a silicon master using top-down nanofabrication tools in cleanroom facilities highlighting the main fabrication steps of the nanofluidic GIE trapping region. (b) Replica molding of the original silicon master using a UV curable resin (OrmoStamp) to obtain a negative master. This step can be repeated unlimited to receive multiple negative masters enabling a highthroughput production of polydimethylsiloxane (PDMS) devices. Each obtained OrmoStamp master can be repeatedly used for PDMS replica molding. (c) Replica molding of the negative OrmoStamp master into PDMS. The cured PDMS devices were plasma activated and covalently bound to a coverslip glass. (d) Sketch of high-throughput fabrication using two-step replica molding. RIE, reactive ion etching.

(MGL-III-532, CNIlaser, Changchun, P.R. China) at $\lambda=532 \mathrm{~nm}$ wavelength. The laser intensity was decreased and controlled using a fixed neutral density (ND) filter of OD 2 and a continuously variable ND filter wheel of OD 0-2 (NDC-50C-2, Thorlabs Inc.). An xy galvo deflection mirror system (GVS002, Thorlabs Inc.) was used to scan the laser over the sample running at $1 \mathrm{kHz}$ rate. The laser was slightly defocused on the back focal plane of an inverted microscope (DMI $5000 \mathrm{M}$, Leica Microsystems) equipped with a $\times 100,1.3$ NA oil-immersion objective (HCX PL FLUOSTAR, Leica Microsystems) and an additional $\times 1.5$ internal tube lens (11 888 699, Leica Microsystems). The reflected and scattered beams were imaged on a CMOS camera (MV-D1024-160-CL-12, Photonfocus AG, 8853 Lachen SZ, Switzerland). To synchronize the camera with the galvo deflection mirror system a four-channel AO-LabView controller (DAQ, National Instruments, Austin, TX, USA) was used and controlled by a custom made LabView software. The images for the residence time measurements were taken at an exposure time of $10 \mathrm{~ms}$ and an acquisition frequency from 5 to $90 \mathrm{~Hz}$ depending on the device geometries and particle sizes scanning a field of view $9 \times 9 \mu \mathrm{m}^{2}$. Images for stiffness measurements were taken at an exposure time of $1 \mathrm{~ms}$ and an acquisition frequency of $111 \mathrm{~Hz}$ scanning a field of view $1 \times 5 \mu \mathrm{m}^{2}$. The lateral trajectories of the particles were obtained by the center of a Gaussian profile fit to each frame, and the axial position is correlated to the amplitude of each profile $\mathrm{fit}^{24,40}$ (Supplementary Information S4). The average lateral localization accuracy in the $x$ - or $y$-direction was $\delta x=6.5 \mathrm{~nm}$ for $d=60 \mathrm{~nm}$ Au NPs and $\delta x=4.5 \mathrm{~nm}$ for $d=80$ and $100 \mathrm{~nm}$ Au NPs.

\section{RESULTS}

\section{Device fabrication}

We used scanning electron microscopy (SEM) to determine whether the shape of the small nanostructures were preserved during the two-step replica molding transfer into OrmoStamp and 
a

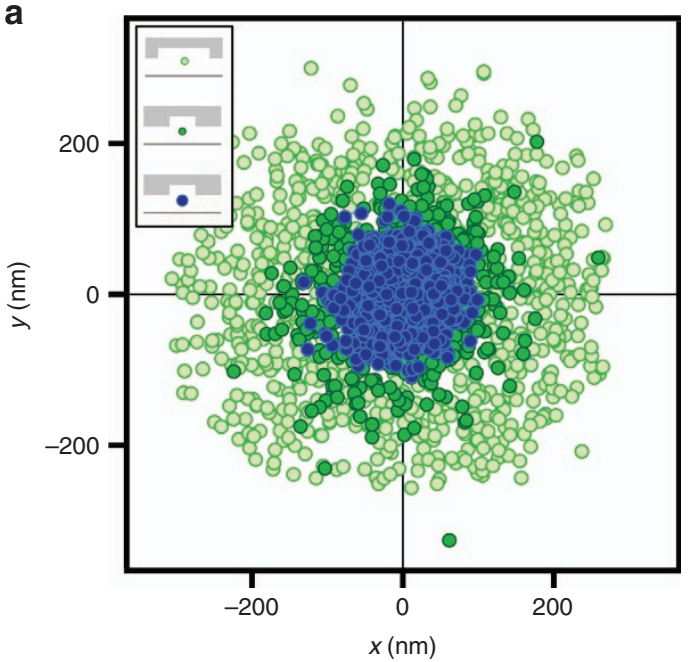

b

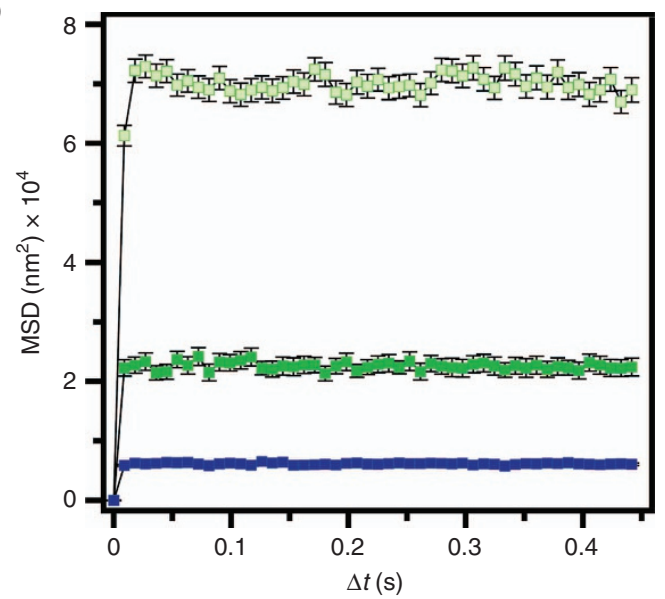

Figure 4 Influence of lateral trap dimensions and particle net charges on the lateral trap stiffness for devices with a channel height of $h_{\mathrm{c}}=160$ and a pocket depth of $h_{\mathrm{p}}=100 \mathrm{~nm}$ (device geometry $G_{2}$ ). (a) Lateral position plots of a $d=60 \mathrm{~nm}$ Au NP trapped by a $w_{\mathrm{p}}=250 \mathrm{~nm}$ (dark green) and a $w_{\mathrm{p}}=500 \mathrm{~nm}$ (light green) circular pocket and a $d=100 \mathrm{~nm}$ (blue) Au NP trapped by $w_{\mathrm{p}}=250 \mathrm{~nm}$ circular pocket. (b) MSD plots corresponding to the lateral position plots in (a). The error bars denote the s.e.m. values.

PDMS. The shapes and dimensions of the 200, 250, and $500 \mathrm{~nm}$ pockets were well resolved in the silicon master as depicted in Figure $3 a$. In the $30^{\circ}$ tilt micrograph, the height difference of the pocket depth $\left(h_{\mathrm{p}}=70 \mathrm{~nm}\right)$ in comparison to the supporting pillar height $\left(h_{\mathrm{c}}=210 \mathrm{~nm}\right)$ is seen. Replica molding into OrmoStamp preserved both lateral and axial dimensions of the nanometersized structures as well as the micrometer-wide channels and supporting pillars (Figure $3 \mathrm{~b}$ ). The flat top of the inverse pockets indicates that the resin could permeate entirely into the pockets before UV curing. To obtain functional devices, besides the device design, proper handling of the PDMS substance by controlling the mixture, curing parameters, and sample filling play critical roles (see fabrication details in Supplementary Information S2). All pocket dimensions could be transferred from OrmoStamp into PDMS as seen in the SEM image of Figure 3c. The lateral dimensions of the pockets and supporting pillars were entirely preserved during the PDMS molding. However, the well-defined axial profile of the pockets and supporting pillars in the OrmoStamp looked smoothened and more shallow in the PDMS mold.

\section{Electrostatic potential landscape}

In GIE-trapping devices, the induced electrostatic potentials depend on a number of parameters, such as the channel and trap height, lateral trap dimension, solution ionic strength and $\mathrm{pH}$, and the surface charge density of the cover glass and PDMS surfaces. Whereas $\mathrm{SiO}_{x}$-based GIE trapping devices have similar material layers on all decisive surface sides, PDMS-based GIE trapping devices consist of a top PDMS surface and a bottom glass surface layer. However, similar surface Zeta potentials of both activated PDMS and activated glass in solution of about $\zeta_{\text {surface }}$ $\sim-80 \mathrm{mV}$ (Supplementary Information S5) indicate similar numbers of spontaneous ionization of silanol groups in water. Thus, it may be expected that the potential minimum in GIE trapping devices made from PDMS and glass substrates results in the slit midplane of the nanofluidic channel.

The trapping strengths of different geometries were characterized by tracking the lateral motion of trapped Au NPs. Exemplary position plots of Au NPs trapped in $G_{2}$ devices $\left(h_{\mathrm{c}}=160 \mathrm{~nm}, h_{\mathrm{p}}=100 \mathrm{~nm}\right)$ with a particle diameter of $d=60 \mathrm{~nm}$ in pockets of $w_{\mathrm{p}}=250$ and $500 \mathrm{~nm}$ and with larger particles of $d=100 \mathrm{~nm}$ in $w_{\mathrm{p}}=250 \mathrm{~nm}$ pockets are shown in Figure $4 \mathrm{a}$. These scatterplots underline the influence of geometrical parameters and particle net charges on the spatial confinement of the particles. As expected, a smaller trap width of $w_{\mathrm{p}}=250 \mathrm{~nm}$ confines the particle to smaller dimensions in comparison to larger $w_{p}=500 \mathrm{~nm}$ ones. Moreover, the $100 \mathrm{~nm}$ particles, carrying a higher net charge of $\sim-258$ e compared to the $\sim-92 e$ of $60 \mathrm{~nm}$ particles, experience a stronger trapping by the pockets with the same diameter (Supplementary Movie 1). The radial symmetry of the scatterplots verifies the high replica mold fabrication quality of the lateral dimensions of the pockets as also shown from SEM inspection (Figure 3c). To quantify the trapping strength, the 2D mean-square displacement (MSD), $<[\Delta r(\Delta t)]^{2}>$, was evaluated as a function of lag time $\Delta t$ for each series of acquired frames for a trapped particle (Figure $4 \mathrm{~b}$ ). For a particle with restricted diffusion, the MSD reaches a plateau for lag times much higher than its relaxation time $\tau_{R}$ in the potential well (the time a non-trapped particle would take to freely diffuse across a distance corresponding to the width of the potential well $)^{41}$. For a harmonic potential, the plateau of the MSD is directly correlated to the radial trap stiffness $k_{\mathrm{r}}$ as ${ }^{18,42}$

$$
<[\Delta r]_{\mathrm{p}}^{2}>=\frac{4 k_{\mathrm{B}} T}{k_{\mathrm{r}}}
$$

where $k_{\mathrm{B}}$ is the Boltzmann constant and $T$ is the absolute temperature. For the $60 \mathrm{~nm}$ particles trapped by the $w_{\mathrm{p}}=500 \mathrm{~nm}$ pockets in a $G_{2}$ device, a radial trap stiffness of $k_{\mathrm{r}}=0.22$ $( \pm 0.06) \mathrm{fN} \mathrm{nm}^{-1}$ was obtained. Decreasing the trap diameter to $w_{\mathrm{p}}=250 \mathrm{~nm}$ confined the motion of the $60 \mathrm{~nm}$ particles stronger and thus increased the trap stiffness to $k_{\mathrm{r}}=0.8( \pm 0.3) \mathrm{fN} \mathrm{nm}^{-1}$, as expected. The $100 \mathrm{~nm}$ particles trapped by the same pockets of $w_{\mathrm{p}}=250 \mathrm{~nm}$ experienced an even stronger trap stiffness of $k_{\mathrm{r}}=2.9( \pm 0.9) \mathrm{fN} \mathrm{nm}^{-1}$ due to their higher net surface charge. We would like to point out that we observed no trap stiffness variation along the width of the nanofluidic channels showing that there is no roof sagging towards the middle of the channel width (Supplementary Information S1).

In addition to the lateral confinement of the electrostatic potentials, we evaluate their depth by measuring the mean residence time $\bar{\tau}_{\mathrm{K}}$ (Kramers time), defined as the average time a particle dwells inside a trap before escaping. For a harmonic potential, the Kramers time is given by

$$
\bar{\tau}_{\mathrm{K}} \cong \tau_{\mathrm{R}} e^{\frac{Q}{k_{\mathrm{B}} T}},
$$

where $k_{\mathrm{B}} T$ is the thermal energy and $Q=q \Delta \psi$ the potential depth with $q$ the surface net charge of the particle and $\Delta \psi$ the electrostatic potential difference between center of the trap and a 


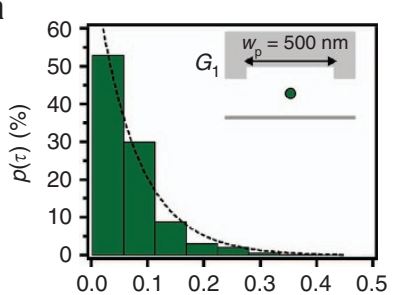

C
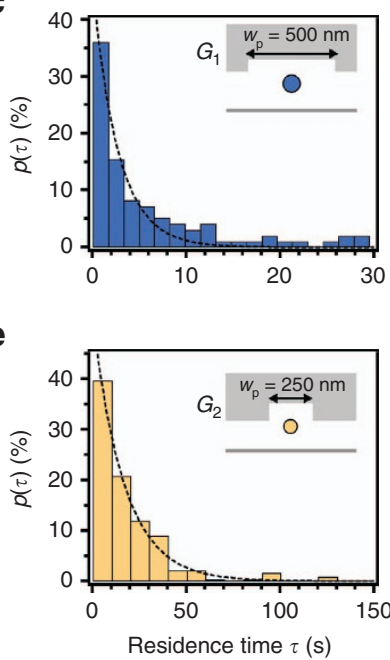

g

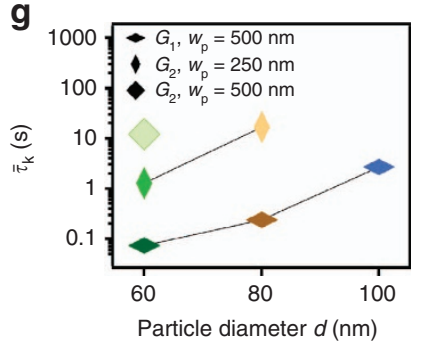

i

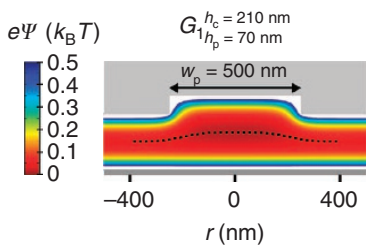

b

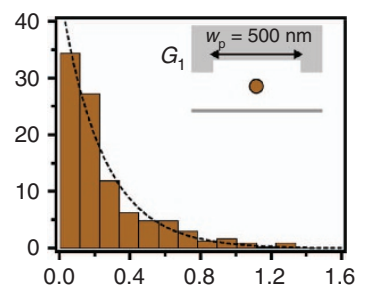

d

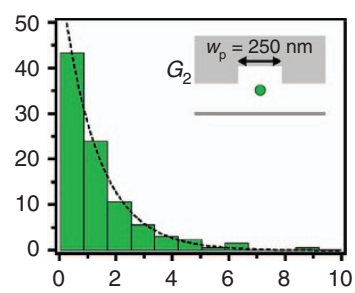

f

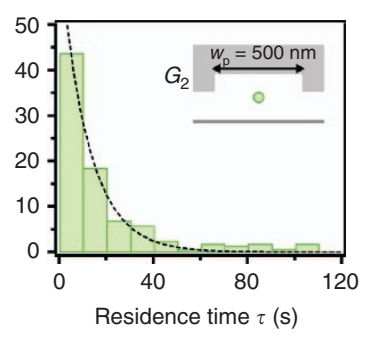

h

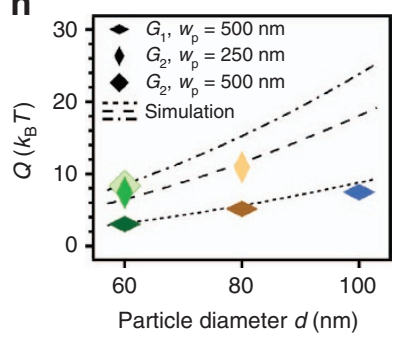

j

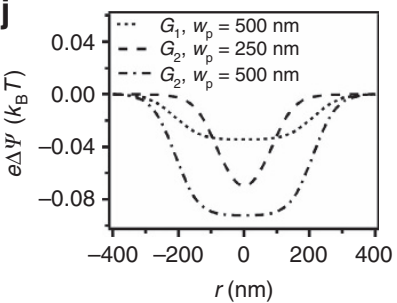

position outside the trap in the nanofluidic channel ${ }^{16,43,44}$. We quantitatively analyzed the mean residence time by monitoring 100-300 escaping events for each particle size in various pocket and device geometries at a monovalent ionic concentration of $c_{0}=0.1 \mathrm{mM}$. In this procedure, $\bar{\tau}_{\mathrm{K}}$ is extracted from the 'residence time probability distribution', $p(\tau)$, which decays exponentially with the residence time $\tau$ of the individual particles as ${ }^{44}$ :

$$
p(\tau)=A e^{-\frac{\tau}{\tau_{K}}} .
$$

Measured $p(\tau)$ are illustrated in Figures $5 a-f$ and the corresponding $\bar{\tau}_{K}$ obtained from these graphs are plotted in Figure $5 \mathrm{~g}$. For different particle sizes trapped in the same geometry of $h_{\mathrm{c}}=210 \mathrm{~nm}, h_{\mathrm{p}}=70 \mathrm{~nm}\left(G_{1}\right)$ and with a pocket width of $w_{\mathrm{p}}=500 \mathrm{~nm}$, smaller particles escape faster from the potential wells. Sixty nanometers Au NPs carrying a net charge of $\sim-92 e$

Figure 5 Residence time measurements. (a-f) Histograms of the residence time probability distribution showing the exponential decay of the residence times of trapped Au NPs in different device geometries (dashed lines are exponential fits) at a concentration of $c_{0}=0.1 \mathrm{mM}$. (a-c) $60 \mathrm{~nm}$ (dark green, a), $80 \mathrm{~nm}$ (dark brown, b), and $100 \mathrm{~nm}$ (blue, c) Au NPs trapped in devices with a nanofluidic channel height of $h_{\mathrm{c}}=210 \mathrm{~nm}$, a pocket depth of $h_{\mathrm{p}}=70 \mathrm{~nm}$ (device geometry $G_{1}$ ) and a width of $w_{p}=500 \mathrm{~nm}, N=278,281$ and 104 trapping events. (d and e) $60 \mathrm{~nm}$ (middle green, d) and $80 \mathrm{~nm}$ (light brown, e) Au NPs trapped in $G_{2} / w_{p}=250 \mathrm{~nm}, N=290$ and 235 trapping events. f) $60 \mathrm{~nm}$ Au NPs trapped in $G_{2} / w_{p}=500 \mathrm{~nm}$, $N=275$ trapping events. (g) Kramers time corresponding to the histogram distributions of (a-f) as a function of particle diameter. (h) Potential depths $Q$ in $k_{\mathrm{B}} T$ as a function of particle diameter calculated from the experimentally obtained Kramers time and from simulations (dashed lines). (i) Simulation of the electrostatic potential of a point charge of $-1 e$ by solving the nonlinear PoissonBoltzmann equation numerically for the device geometry $G_{1}$ and a pocket width of $w_{p}=500 \mathrm{~nm}$. (j) Extraction of the electrostatic potential difference of a point charge of $-1 e$ for the device geometry $G_{1} / w_{p}=500 \mathrm{~nm}, G_{2} / w_{p}=250 \mathrm{~nm}$ and $G_{2} / w_{p}=500 \mathrm{~nm}$ as a function of $r$ along the axial energy minimum (black dashed line in i).

quickly escaped from the traps with a very short Kramers time of $\bar{\tau}_{\mathrm{K}}=0.073( \pm 0.012) \mathrm{s}$. By increasing the diameter of the particles to $80 \mathrm{~nm}$, carrying a higher net charge of $\sim-168 e$, the Kramers time was increased to $\bar{\tau}_{\mathrm{K}}=0.242( \pm 0.037) \mathrm{s}$ and further to $\bar{\tau}_{\mathrm{K}}=2.70$ $( \pm 0.36) \mathrm{s}$ for $100 \mathrm{~nm}$ gold particles with a net charge of $\sim-258 \mathrm{e}$. Experimentally, we find an over-exponential increase of the Kramers time as a function of particle diameter. To obtain the potential depths

$$
Q \cong \ln \left(\frac{\bar{\tau}_{\mathrm{K}}}{\tau_{\mathrm{R}}}\right) k_{\mathrm{B}} T
$$

of each system, the relaxation time $\tau_{R}$ was determined by the experimentally measured trap stiffness $k_{\mathrm{r}}$ and the Diffusion coefficients $D$ of the particles (Table 1 ). In a harmonic potential, $\tau_{\mathrm{R}}$ is related to the trap stiffness $k_{\mathrm{r}}$ and the Diffusion coefficients $D$ of the particles as ${ }^{18,45}$

$$
\tau_{\mathrm{R}}=\frac{k_{\mathrm{B}} T}{D k_{\mathrm{r}}},
$$

where $D=k_{\mathrm{B}} T / 3 \pi \eta d$ for a particle with diameter $d$ in a solution of dynamic viscosity $\eta$.

For the $60 \mathrm{~nm}$ Au NPs trapped in the $G_{1}$ devices, only a potential depth of about $Q \cong 3.0 k_{B} T$ is required to be released from the $500 \mathrm{~nm}$ pockets, explaining the fast escape of the particles from the trap. The potential depth is increased to $Q \cong 5.2 k_{\mathrm{B}} T$ and $Q \cong 7.5 k_{\mathrm{B}} T$ for the 80 and $100 \mathrm{~nm}$ Au NPs carrying a higher net charge. From simulating the electrostatic potential of a point charge by solving the nonlinear Poisson-Boltzmann equation numerically ${ }^{16}$ (COMSOL Multiphysics 4.2, see Figures $5 i$ and $j$ and Supplementary Information S6) we can extract the potential depths for the given geometry as a function of the particle size (dashed lines in Figure $5 \mathrm{~h}$ ). For the simulations, a mean surface charge density of the particles was taken from measurements of $\sigma_{\mathrm{p}}=8 \times 10^{-3} \mathrm{enm}^{-2}$ and a surface charge density of the substrate glass and PDMS were estimated from spontaneous ionization in water of about $\sigma_{\mathrm{s}} \sim 3 \times 10^{-3} \mathrm{e} \mathrm{nm}{ }^{-2}$ (Ref. 46). The experimental results and the good agreement with the simulations show that the Kramers time increases over-exponentially with the particle diameter. Taking equation (2) and equation (5), this can be confirmed by the proportionality of $\bar{\tau}_{\mathrm{K}}$ as

$$
\bar{\tau}_{\mathrm{K}} \cong \frac{k_{\mathrm{B}} T}{D k_{\mathrm{r}}} e^{\frac{q \Delta \psi}{k_{\mathrm{B}} T}} \propto \frac{e^{a d^{2}}}{d},
$$


Table 1 Relaxation times $\tau_{\mathrm{R}}$ for 60,80 , and $100 \mathrm{~nm}$ gold particles in different trap geometries

\begin{tabular}{|c|c|c|c|c|c|c|}
\hline $\begin{array}{l}\text { Device } \\
\text { design }\end{array}$ & $\begin{array}{l}\text { Device geometry } \\
\qquad h_{\mathrm{c}}, h_{\mathrm{p}}(\mathrm{nm})\end{array}$ & $\begin{array}{c}\text { Trap diameter } \\
W_{\mathrm{p}}(\mathrm{nm})\end{array}$ & $\begin{array}{l}\text { Particle diameter } \\
\qquad d(\mathrm{~nm})\end{array}$ & $\begin{array}{l}\text { Diffusion coefficient } \\
\qquad D\left(\mu \mathrm{m}^{2} \mathrm{~s}^{-1}\right)\end{array}$ & 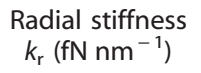 & $\begin{array}{c}\text { Relaxation time } \\
\tau_{\mathrm{R}}(\mathrm{ms})\end{array}$ \\
\hline$G_{1}$ & 210,70 & 500 & 60 & 6.38 & 0.18 & 3.5 \\
\hline \multirow[t]{3}{*}{$G_{2}$} & 160,100 & 250 & 60 & 6.38 & 0.81 & 0.8 \\
\hline & & 250 & 80 & 5.23 & 2.61 & 0.3 \\
\hline & & 500 & 60 & 6.38 & 0.22 & 2.9 \\
\hline
\end{tabular}

The diffusion coefficients $D$ were measured using a dynamic light scattering system.

assuming the net surface charge $q$ of the particles is proportional to $d^{2}$, the diffusion coefficients $D$ inverse proportional to $d$, and the stiffness $k_{r}$ proportional to $d^{2}\left(q \Delta \psi(r)=k_{r} r^{2} / 2\right)^{21}$. A stable trapping longer than a few seconds in geometry $G_{1}\left(h_{c}=210 \mathrm{~nm}\right.$ and $w_{\mathrm{p}}=70 \mathrm{~nm}$ ) devices and at an ionic concentration of $c_{0}=0.1 \mathrm{mM}$ was only possible for the $100 \mathrm{~nm}$ particles. For creating deeper potential wells, the height $h_{\mathrm{c}}$ of the nanofluidic channel and the depth $h_{\mathrm{p}}$ and width $w_{\mathrm{p}}$ of the pockets are important geometrical parameters to vary. Decreasing the channel height results in deeper potentials and thus longer trapping times of the particles as well as increasing of the potential depth.

By fabricating a second design of GIE trapping devices with a reduced nanofluidic channel height of $h_{\mathrm{c}}=160 \mathrm{~nm}$ and slightly deeper pockets of $h_{\mathrm{p}}=100 \mathrm{~nm}$ (device geometry $G_{2}$ ), 60 to $100 \mathrm{~nm}$ particles could be stably trapped by the pockets as seen in the iSCAT images of Figure $1 \mathrm{~b}$. The residence time probability distribution plots for the $60 \mathrm{~nm}$ Au NPs trapped in pocket widths of $w_{\mathrm{p}}=250 \mathrm{~nm}$ and $500 \mathrm{~nm}$ and for the $80 \mathrm{~nm}$ Au NPs trapped in a pocket width of $w_{p}=250 \mathrm{~nm}$ are shown in Figures $5 \mathrm{~d}-\mathrm{f}$. For the $60 \mathrm{~nm}$ and $80 \mathrm{~nm}$ particles trapped in the $w_{\mathrm{p}}=250 \mathrm{~nm}$ pockets, Kramer times of $\bar{\tau}_{\mathrm{K}}=1.28( \pm 0.05) \mathrm{s}$ and $\bar{\tau}_{\mathrm{K}}=16.8( \pm 0.3) \mathrm{s}$ were obtained with a corresponding potential depth of $Q \cong 7.4 k_{\mathrm{B}} T$ and $Q \cong 10.9 k_{\mathrm{B}} T$. Trapping the $60 \mathrm{~nm}$ particles in $w_{\mathrm{p}}=500 \mathrm{~nm}$ pockets (same pocket width as for the device geometry $G_{1}$ ), the Kramers time was increased to $\bar{\tau}_{K}=12.2( \pm 0.3) \mathrm{s}$ resulting in a potential depth of $Q \cong 8.3 k_{\mathrm{B}} T$. For the same pocket width, this is a $165 \times$ increase of the Kramers time compared to the $\bar{\tau}_{\mathrm{K}}=0.073( \pm 0.012) \mathrm{s}$ measured in the $G_{1}$ devices. The experimental observations confirm that particles trapped in pockets with a larger diameter have longer trapping times caused by a deeper potential (up to a certain limit) and lower counts per time of hitting the potential boundaries, due to the longer relaxation times within the larger pockets. The quantitative analysis of the Kramers time and potential depth requires statistics of many escaping events. Since for the second device design $G_{2}$, the $80 \mathrm{~nm}$ particles in the $w_{\mathrm{p}}=500 \mathrm{~nm}$ pockets and the $100 \mathrm{~nm}$ particles in the $w_{\mathrm{p}}=250 \mathrm{~nm}$ and $500 \mathrm{~nm}$ pockets were stably trapped for several minutes to days the corresponding potential depths could be obtained only by simulations. For the $100 \mathrm{~nm}$ particles trapped by the $W_{\mathrm{p}}=250 \mathrm{~nm}$ pockets in a $G_{2}$ device, a potential depth of $Q \sim 18 k_{\mathrm{B}} T$ can be estimated which corresponds to a Kramers time of about $\bar{\tau}_{\mathrm{K}} \sim 10 \mathrm{~h}$. When trapped in the $w_{\mathrm{p}}=500 \mathrm{~nm}$ pockets, potential depths of $Q \sim 24 k_{\mathrm{B}} T$ are calculated resulting in an extremely long Kramers time beyond experimental demands.

\section{Tunable trapping by elastic deformation}

In contrast to rigid materials, such as silicon and glass, PDMS is an elastomer that can be compressed and bent easily ${ }^{47}$. This provides new advantages in addition to its low-cost fabrication as discussed in previous sections. We use this unique feature of our nanofluidic system to tune the channel heights $h_{c}$ and hence the trap stiffness and potential depth. Compressing the PDMS part of the devices by using mechanical forces, modifies the nanofluidic channel height and thus allows for an additional in situ tuning of the trap stiffness and residence times. By applying a compression pressure on the PDMS, valves and pumps in devices have been realized by, for example, pneumatic pressure ${ }^{48-50}$, torque actuation from embedded screws ${ }^{51}$ or solenoids ${ }^{52}$ to open and close microfluidic channels.

To apply a mechanical deformation, a precision screw and a silicon plate were used as sketched in Figures $6 a$ and $b$. The compression distance and the elastic modulus of the PDMS of about $E=3.6 \mathrm{MPa}$ (Ref. 30) was used to quantify the pressure exerted on the device. The result for an individual $100 \mathrm{~nm}$ Au NP trapped in a $w_{\mathrm{p}}=250 \mathrm{~nm}$ pocket at an initial nanofluidic channel height of $h_{\mathrm{c}, 0}=160 \mathrm{~nm}$ and a pocket depth of $h_{\mathrm{p}}=100 \mathrm{~nm}$ (device geometry $G_{2}$ ) is shown in Figure $6 \mathrm{c}$. If no compression pressure was applied on the PDMS device $(P=0 \mathrm{kPa})$, a radial stiffness of $k_{\mathrm{r}, 0}=3 \times 10^{-3} \mathrm{pN} \mathrm{nm} \mathrm{n}^{-1}$ was measured. Applying a stepwise deformation pressure on the PDMS device of about $\Delta P \sim 10 \mathrm{kPa}$ each step, resulted in the reduction of the nanofluidic channel height of about $\Delta h_{c}(\Delta P) \sim 25 \mathrm{~nm}$ and thus to a stronger trap stiffness. The nanofluidic channel heights were derived by estimating the radial trap stiffness $k_{\mathrm{r}, \text { sim }}$ for different simulated channel heights according to $q \Delta \psi=k_{\mathrm{r}, \operatorname{sim}} r^{2} / 2$ as shown in Figure $6 \mathrm{~d}$ and comparing $k_{\mathrm{r} \text {,sim }}$ with the experimentally obtained trap stiffness (Figure $6 \mathrm{e}$ ). Here, $q \Delta \psi$ represents the electrostatic energy for a point charge $q$ of -258 e. During the approach (reduction of channel height), the particle was further laterally and axially confined, which can be seen in the decrease of the radial displacement and the corresponding MSDs in Figure $6 c$, the Supplementary Information S4, and the Supplementary Movie 2. At $P=50 \mathrm{kPa}$, the radial trap stiffness increased $45 \times$ to $k_{\mathrm{r}, 50}=0.09 \mathrm{pN} \mathrm{nm}^{-1}$. At this trap stiffness, corresponding to a channel height of less than $40 \mathrm{~nm}$, a potential depth of more than $Q_{\max } \sim 200 k_{\mathrm{B}} T$ was obtained from our simulations. Additionally, since a smaller nanofluidic channel height than the actual particle diameter was estimated, the particle could be trapped electrostatically as well as geometrically, a remarkable advantage compared to chip-based devices made from rigid materials. This process could be reversed, proving the contact-free nature of the trapping method, by releasing the pressure and going back to $P=0 \mathrm{kPa}$, resulting in a less confined particle with the initial radial trap stiffness of about $k_{\mathrm{r}, 0}=3 \times 10^{-3} \mathrm{pN} \mathrm{nm}{ }^{-1}$ (Figures $6 \mathrm{c}$ and e).

\section{DISCUSSION}

Our results demonstrate that deeper potential depths as the required of $10 k_{\mathrm{B}} T$ for stable trapping can be obtained by GIE trapping in soft matter devices. For potential depths $<10 k_{\mathrm{B}} T$ trapping times of only milliseconds to some seconds were observed, whereas reliable trapping of Au NPs was achieved from several seconds up to hours and days if the potential depths exceeded $10 k_{\mathrm{B}} T$. Furthermore, the unique feature of soft PDMS devices to manipulate the nanofluidic channel height by an 
a

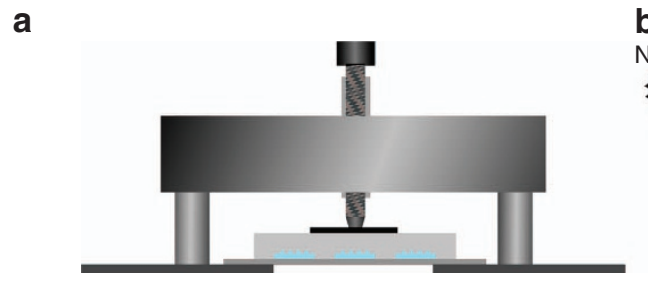

b

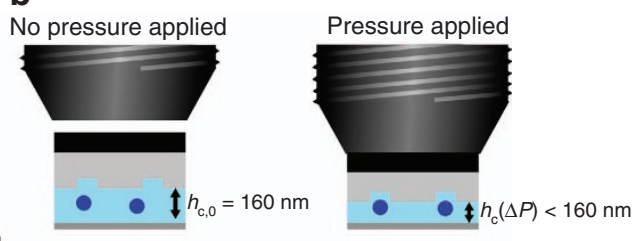

C
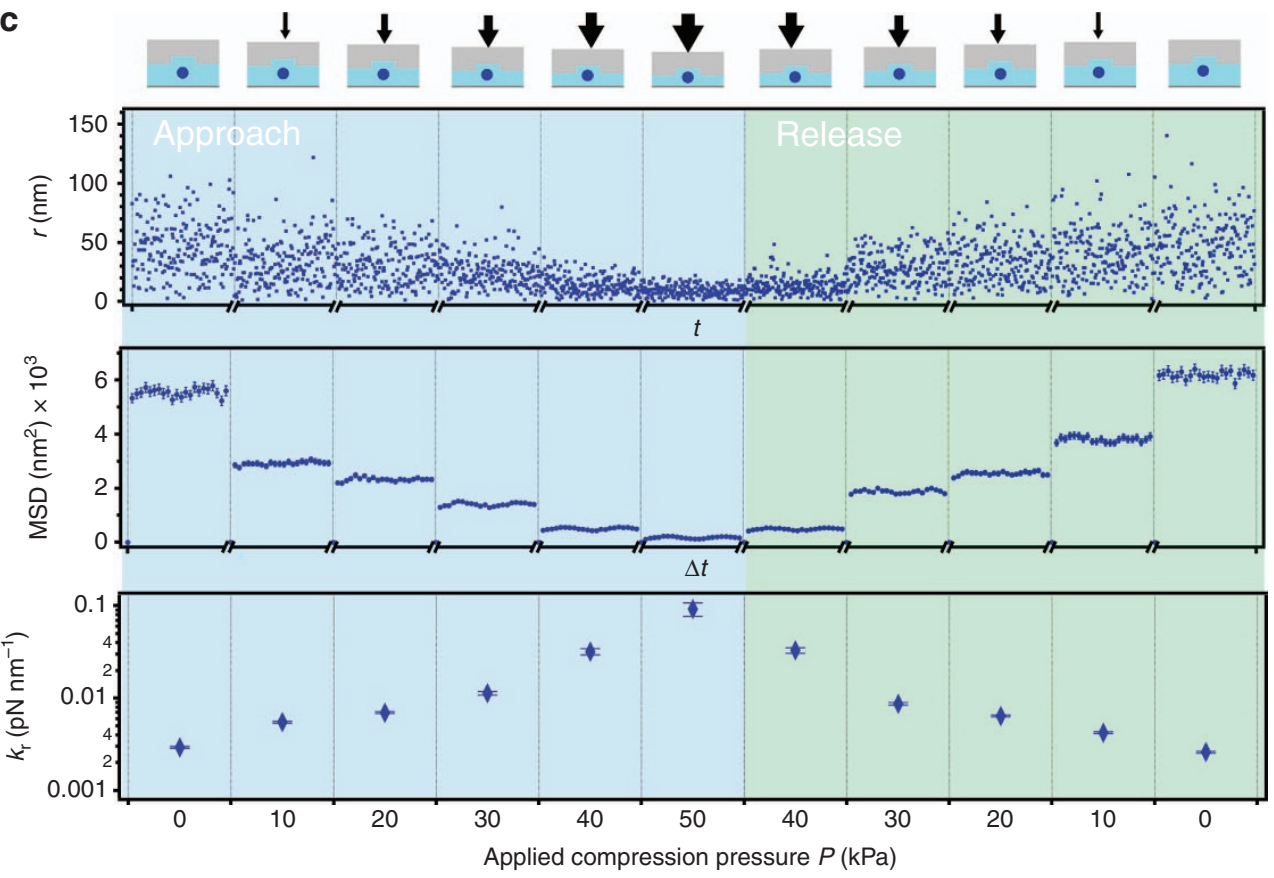

d

e

$P(\mathrm{kPa})$
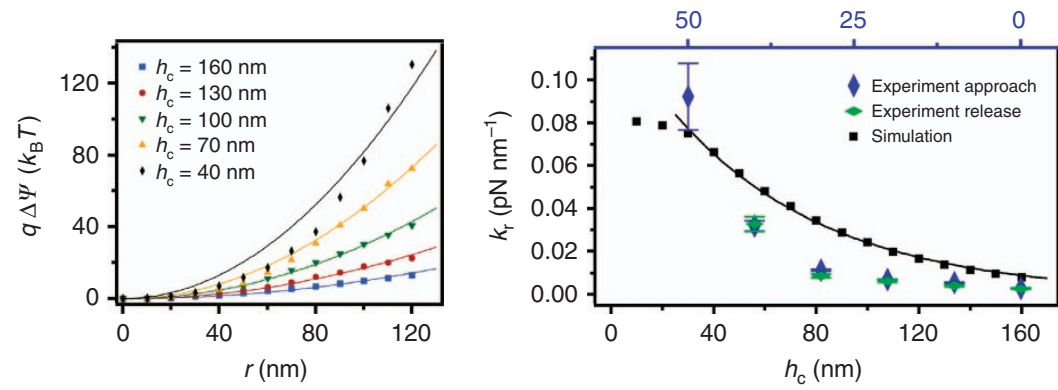

Figure 6 Active manipulation of trapping performance by elastic deformation of the polydimethylsiloxane (PDMS) device. (a) Sketch of the experimental setup using a precision screw to apply an axial compression force onto the PDMS device. (b) Magnified area of (a), illustrating the height change of the nanofluidic channel when a compression force is applied to the device. (c) Active manipulation of the trapping strength of a $d=100 \mathrm{~nm}$ Au NP trapped in a $w_{\mathrm{p}}=250 \mathrm{~nm}$ circular pocket at an initial nanofluidic channel height of $h_{\mathrm{c}, 0}=160 \mathrm{~nm}$ and a pocket depth of $h_{\mathrm{p}}=100 \mathrm{~nm}$. In the top graph the radial fluctuations $r$ of the particle are plotted as a function of time $t$ at different applied pressures. The corresponding MSDs are shown in the middle graph as a function of the lag time $\Delta t$. The obtained radial trap stiffnesses from the MSDs are plotted in the lower graph as a function of the applied compression pressure $P$. Increasing the compression pressure of to the PDMS results in a nanofluidic channel height reduction and thus higher trapping strength. (d) Radial trap stiffness fits on simulations of the electrostatic energy along the axial energy minimum for a point charge of $q=-258$ e (100 nm particle) for different nanofluidic channel heights $h_{\mathrm{c}}$ (e) Comparison of measured and simulated values of the radial trap stiffness $k_{\mathrm{r}}$ as a function of applied compression pressure $P$ and nanofluidic channel height $h_{\mathrm{c}}$.

applied pressure enables straightforward tuning of the chip performance during the experiment and thus opens the capability of active trapping and releasing of nanoparticles. The performance of current chip-based GIE trapping devices made from rigid $\mathrm{SiO}_{\mathrm{x}}$ materials however is characterized by their initial fabricated geometric parameters, especially the nanofluidic channel height. In addition, achieving trapping potentials of more than
$Q_{\max } \sim 200 k_{\mathrm{B}} T$ and having the possibility of trapping the Au NPs geometrically could make the trapping of smaller nano-objects in physiological buffer conditions possible. Tuning the nanofluidic channel height during the experiments gives the possibility to load a particle solution without clogging, followed by trapping the particles by an applied pressure while still having the option to change the condition of the solution by an integrated flow fluidic 
system or by diffusion. Krishnan reported that even uncharged particles might be trapped in the nanofluidic indentations within potential depths $>10 k_{\mathrm{B}} T$, if the ratio of the particle diameter and the nanofluidic channel height $d / h_{c}$ is larger than about 0.6 (Ref. 53). This effect was explained by the repelling of the particle from the nanofluidic channel into the trap caused by the counterions entropy of the charged channel walls. Thus, the feasibility to manipulate the nanofluidic channel heights during the experiment down to the size of the particle diameters may extend the PDMS-based trapping method for trapping even uncharged particles. For nanofluidic channel heights smaller than $\sim 50 \mathrm{~nm}$, the simulated electrostatic energies scale slightly different from the predicted correlation $q \Delta \psi=k_{\mathrm{r}, \mathrm{sim}} r^{2} / 2$ (Figure $6 \mathrm{~d}$ ). For such small channel heights, the potential energy landscape might deviate from a perfect harmonic potential, caused by the reduced axial dimension but still constant lateral dimension of the trap width. In addition, the simulated trapping strengths deviate from our experimentally explored stiffnesses as seen in Figure 6e. We note that for the simulations no external fit parameters were used and that the simulations are based on the obtained mean values, described in section 3.2. The experiment in Figure 6 demonstrates the behavior of an individually trapped $\mathrm{Au}$ NP. Thus, the uncertainties in the particle size and charge and in the correlation between the applied pressure and nanofluidic channel height are the main reasons for this deviation. At small channel heights further effects such as the finite size of the particle and the effect of entropic trapping play an additional role. These effects could be studied in future experiments by exploring the variation of each effect individually.

From the viewpoint of implementation, such systems can have a great impact in the field of nanofluidics since the flexibility and low cost of fabrication bypasses the need for cleanroom facility for top-down processes. Moreover, the present method is integrated with the well-established microfluidic techniques and infrastructure, enabling the integration of GIE trapping nanofluidic devices with more complex fluidic systems such as particle sorting or trapping along concentration gradients ${ }^{23}$. Demonstrating that GIE trapping devices can be fabricated from replica molding processes opens additionally the possibility for fabricating such systems out of a variety of new materials. As an example, GIE trapping devices made from polystyrene foils and UV-curable adhesives could be used for X-ray scattering such as free-electron-laser studies ${ }^{54,55}$.

\section{CONCLUSIONS}

We present nanofluidic trapping devices made from the elastomeric material PDMS for high-throughput fabrication and high-performance contact-free passive trapping of single charged nano-objects. These devices consist of fluidic channels that are $\sim 160 \mathrm{~nm}$ in height but several micrometers in width, enabling the trapping of multiple single particles in parallel by fabricating trap lattices within the channels. Analyzing the lateral motion and residence times of the particles, we could obtain both, the trap strength and the potential depths of our traps experimentally supported by simulations. For the as-fabricated device geometries (that is, without exerted pressure), we found potentials of the electrostatic traps as deep as $Q \cong 24 k_{\mathrm{B}} T$, corresponding to stable trapping times of many days. We were able to actively tune the nanofluidic channel heights by applying a mechanical compression pressure and thus varying the trap stiffness and potential depths in situ. With this feature that is not possible in rigid $\mathrm{SiO}_{\mathrm{x}^{-}}$ based devices, remarkable deep potentials of $Q_{\max } \sim 200 \mathrm{k}_{\mathrm{B}} T$ and high trap stiffness of more than $k_{\mathrm{r}, \max }=0.09 \mathrm{pN} \mathrm{nm}^{-1}$ were achieved. Realizing such high potential depths could facilitate practical implementation of trapping devices for in situ isolation of fundamental biological entities such as macromolecules in physiological buffer conditions. Due to its ease of fabrication, our method opens the feasibility to carry out single and label-free particle research using the GIE trapping method with little effort.

\section{ACKNOWLEDGEMENTS}

This work was funded by the Swiss Nanoscience Institute in Basel, Switzerland (SNI $\mathrm{PhD}$ graduate school, Project P1202). We would like to thank the technical staff of the Laboratory for Micro- and Nanotechnology at the Paul Scherrer Institut, Switzerland. Further, we want to thank Celestino Padeste and Victor Cardaso for their technical help and fruitful discussions.

\section{COMPETING INTERESTS}

The authors declare no conflict of interest.

\section{REFERENCES}

1 Chin CD, Linder V, Sia SK. Commercialization of microfluidic point-of-care diagnostic devices. Lab on a Chip 2012; 12: 2118-2134.

2 Kovarik ML, Gach PC, Ornoff DM et al. Micro total analysis systems for cell biology and biochemical assays. Analytical Chemistry 2012; 84: 516-540.

3 Neuži P, Giselbrecht S, Länge $K$ et al. Revisiting lab-on-a-chip technology for drug discovery. Nature Reviews Drug Discovery 2012; 11: 620-632.

4 Schmidt $\mathrm{H}$, Hawkins AR. The photonic integration of non-solid media using optofluidics. Nature Photonics 2011; 5: 598-604.

5 Nge PN, Rogers Cl, Woolley AT. Advances in microfluidic materials, functions, integration, and applications. Chemical Reviews 2013; 113: 2550-2583.

6 Duan C, Wang W, Xie Q. Review article: Fabrication of nanofluidic devices. Biomicrofluidics 2013; 7: 26501.

7 Wu C, Lin TG, Zhan Z et al. Fabrication of all-transparent polymer-based and encapsulated nanofluidic devices using nano-indentation lithography. Microsystems \& Nanoengineering 2017; 3: 1-9.

8 Chung S, Lee $\mathrm{JH}$, Moon MW et al. Non-lithographic wrinkle nanochannels for protein preconcentration. Advanced Materials 2008; 20: 3011-3016.

9 Huh D, Mills KL, Zhu X et al. Tuneable elastomeric nanochannels for nanofluidic manipulation. Nature Materials 2007; 6: 424-428.

10 Park S-M, Huh YS, Craighead HG et al. A method for nanofluidic device prototyping using elastomeric collapse. Proceedings of the National Academy of Sciences of the United States of America 2009; 106: 15549-15554.

$11 \mathrm{Li} \mathrm{T}$, Kheifets S, Medellin D et al. Measurement of the instantaneous velocity of a Brownian particle. Science 2010; 328: 1673-1675.

12 Kheifets S, Simha A, Melin K et al. Observation of Brownian motion in liquids at short times: Instantaneous velocity and memory loss. Science 2014; 343: 1493-1496.

13 Franosch T, Grimm M, Belushkin $M$ et al. Resonances arising from hydrodynamic memory in Brownian motion-The colour of thermal noise. Nature 2011; 478: 8-11.

14 Ashkin A, Dziedzic JM, Bjorkholm JE et al. Observation of a single-beam gradient force optical trap for dielectric particles. Optics Letters 1986; 11: 288-290.

15 Juan ML, Righini M, Quidant R. Plasmon nano-optical tweezers. Nature Photonics 2011; 5: 349-356.

16 Krishnan M, Mojarad N, Kukura P et al. Geometry-induced electrostatic trapping of nanometric objects in a fluid. Nature 2010; 467: 692-695.

17 Celebrano M, Rosman C, Sönnichsen C et al. Angular trapping of anisometric nano-objects in a fluid. Nano Letters 2012; 12: 5791-5796.

18 Mojarad N, Krishnan M. Measuring the size and charge of single nanoscale objects in solution using an electrostatic fluidic trap. Nature Nanotechnology 2012; 7: 448-452.

19 Gerspach MA, Mojarad N, Pfohl T et al. Glass-based geometry-induced electrostatic trapping devices for improved scattering contrast imaging of nano-objects. Microelectronic Engineering 2015; 145: 43-48.

20 Sharma D, Gerspach MA, Pfohl T et al. Single positively charged particle trapping in nanofluidic systems. Microelectronic Engineering 2017; 175: 43-49.

21 Tae Kim J, Spindler S, Sandoghdar V. Scanning-aperture trapping and manipulation of single charged nanoparticles. Nature Communications 2014; 5: 1-6.

22 Myers CJ, Celebrano M, Krishnan M. Information storage and retrieval in a single levitating colloidal particle. Nature Nanotechnology 2015; 10: 886-891.

23 Gerspach MA, Mojarad N, Sharma D et al. Nanofluidic lab-on-a-chip trapping devices for screening electrostatics in concentration gradients. Microelectronic Engineering 2017; 175: 17-22.

24 Mojarad N, Sandoghdar V, Krishnan M. Measuring three-dimensional interaction potentials using optical interference. Optics Express 2013; 21: 9377-9389.

25 Schift H, Spreu C, Saidani M et al. Transparent hybrid polymer stamp copies with sub-50-nm resolution for thermal and UV-nanoimprint lithography. Journal of 
Vacuum Science \& Technology B: Microelectronics and Nanometer Structures Processing, Measurement, and Phenomena 2009; 27: 2846-2849.

26 Schift H, Spreu C, Schleunitz A et al. Easy mask-mold fabrication for combined nanoimprint and photolithography. Journal of Vacuum Science \& Technology B: Microelectronics and Nanometer Structures Processing, Measurement, and Phenomena 2009; 27: 2850-2853.

27 Cadarso VJ, Kiefer T, Auzelyte V et al. Direct imprinting of organic-inorganic hybrid materials into high aspect ratio sub-100 nm structures. Microsystem Technologies 2014; 20: 1961-1966.

28 Huang YY, Zhou W, Hsia KJ et al. Stamp collapse in soft lithography. Langmuir 2005; 21: 8058-8068.

29 Lee J, Yun YK, Kim Y et al. PDMS nanoslits without roof collapse. Bulletin of the Korean Chemical Society 2009; 30: 1793-1797.

30 Wang Z, Volinsky AA, Gallant ND. Crosslinking effect on polydimethylsiloxane elastic modulus measured by custom-built compression instrument. Journal of Applied Polymer Science 2014; 41050: 1-4.

31 Kim J-J, Lee Y, Kim HG et al. Biologically inspired LED lens from cuticular nanostructures of firefly lantern. Proceedings of the National Academy of Sciences of the United States of America 2012; 109: 18674-18678.

32 Bender M, Plachetka U, Ran J et al. High resolution lithography with PDMS molds. Journal of Vacuum Science \& Technology B: Microelectronics and Nanometer Structures 2004; 22: 3229-3232.

33 Loeb AL, Overbeek JTG, Wiersema PH. The electrical double layer around a spherical colloid particle: computation of the potential, charge density, and free energy of the electrical double layer around a spherical colloid particle. Massachusetts Institute of Technology. 1961.

34 Israelachvili JN. Intermolecular and Surface Forces, Third Edition. Intermolecular and Surface Forces. Academic Press. 2010.

35 Kalka H. Electrical conductivity (EC). aqion 2015. Available at http://www.aqion.de/ site/130 (Accessed: 20th June 2017).

36 Kukura P, Ewers $\mathrm{H}$, Müller $\mathrm{C}$ et al. High-speed nanoscopic tracking of the position and orientation of a single virus. Nature Methods 2009; 6: 923-927.

37 Mojarad NM, Zumofen G, Sandoghdar V et al. Metal nanoparticles in strongly confined beams: Transmission, reflection and absorption. Journal of the European Optical Society: Rapid Publications 2009; 4: 1-6.

38 Jacobsen $\mathrm{V}$, Stoller $\mathrm{P}$, Brunner $\mathrm{C}$ et al. Interferometric optical detection and tracking of very small gold nanoparticles at a water-glass interface. Optics Express 2006; 14: 405-414.

39 Ortega-Arroyo J, Kukura P. Interferometric scattering microscopy (iSCAT): new frontiers in ultrafast and ultrasensitive optical microscopy. Physical Chemistry Chemical Physics 2012; 14: 15625-15636.

40 Fringes S, Skaug M, Knoll AW. In situ contrast calibration to determine the height of individual diffusing nanoparticles in a tunable confinement. Journal of Applied Physics 2016; 119: 1-28.

41 Kusumi A, Sako Y, Yamamoto M. Confined lateral diffusion of membrane receptors as studied by single particle tracking (nanovid microscopy). Effects of calcium-induced differentiation in cultured epithelial cells. Biophysical Journal 1993; 65: 2021-2040.

42 Tóthová J, Vasziová G, Glod L et al. Langevin theory of anomalous Brownian motion made simple. European Journal of Physics 2011; 32: 645-655.

43 Kramers HA. Brownian motion in a field of force and the diffusion model of chemical reactions. Physica 1940; 7: 284-304.

44 Libchaber A, Simon A. Escape and synchonization of a brownian particle. The American Physical Society 1992; 68: 3375-3378.

45 Howard J. Mechanics of Motor Proteins and the Cytoskeleton. Sinauer Associates. Springer Berlin Heidelberg. 2001

46 Behrens SH, Grier DG. The charge of glass and silica surfaces. The Journal of Chemical Physics 2001; 115: 6716-6721.

47 Whitesides G, Stroock A. Flexible methods for microfluidics. Physics Today 2001; 54: 42-48.

48 Unger MA, Chou HP, Thorsen T et al. Monolithic microfabricated valves and pumps by multilayer soft lithography. Science 2000; 288: 113-116.

49 Hong JW, Quake SR. Integrated nanoliter systems. Nature Biotechnology 2003; 21: 1179-1183.

50 Thorsen T, Maerkl SJ, Quake SR. Microfluidic large-scale integration. Science 2002; 298: $580-584$.

51 Weibel DB, Kruithof $M$, Potenta $S$ et al. Torque-actuated valves for microfluidics. Analytical Chemistry 2005; 77: 4726-4733.

52 Hulme SE, Shevkoplyas SS, Whitesides GM. Incorporation of prefabricated screw, pneumatic, and solenoid valves into microfluidic devices. Lab on a chip 2009; 9 : 79-86.

53 Krishnan M. Electrostatic free energy for a confined nanoscale object in a fluid. The Journal of Chemical Physics 2013; 138.

54 Brennich ME, Nolting J-F, Dammann C et al. Dynamics of intermediate filament assembly followed in micro-flow by small angle X-ray scattering. Lab on a chip 2011; 11: 708-716.

55 Urbani R, Westermeier F, Banusch B et al. Brownian and advective dynamics in microflow studied by coherent X-ray scattering experiments. Journal of Synchrotron Radiation 2016; 23: 1401-1408.

(i) This work is licensed under a Creative Commons Attribution 4.0 International License. The images or other third party material in this article are included in the article's Creative Commons license, unless indicated otherwise in the credit line; if the material is not included under the Creative Commons license, users will need to obtain permission from the license holder to reproduce the material. To view a copy of this license, visit http://creativecommons.org/licenses/ by/4.0/

(c) The Author(s) 2017

Supplementary information for this article can be found on the Microsystems \& Nanoengineering website: http://www.nature.com/ micronano. 